\title{
Using Angle of Arrival (Bearing) Information in Network Localization
}

\author{
Tolga Eren, Walter Whiteley and Peter N. Belhumeur
}

\begin{abstract}
In this paper, we consider using angle of arrival information (bearing) for network localization and control in two different fields of multi-agent systems: (i) wireless sensor networks; (ii) robot networks. The essential property we require in this paper is that a node can infer heading information from its neighbors. We address the uniqueness of network localization solutions by the theory of globally rigid graphs. We show that while the parallel rigidity problem for formations with bearings is isomorphic to the distance case, the global rigidity of the formation is simpler (in fact identical to the simpler rigidity case) for a network with bearings, compared to formations with distances. We provide the conditions of localization for networks in which the neighbor relationship is not necessarily symmetric.
\end{abstract}

\section{INTRODUCTION}

Network localization is a basic service of many emerging computing/networking paradigms. It is typically required for wireless sensors and robotic agents for monitoring the environment or for surveillance, or for routing packets using geometric-aware routing. In pervasive computing, knowing the locations of the computers and the printers in a building will allow a computer to send a printing job to the nearest printer. The aim of localization is to assign geographic coordinates to each node in the sensor network. In this paper, nodes are thought of as sensor nodes in sensor networks or robotic agents in robot formations. The locations may be computed relatively with respect to one another, with unknown translation and rotation, yielding a relative localization; or, the locations may be computed with respect to a global coordinate system, producing an absolute localization. In wireless sensor networks, localization of sensors is a key enabling technology, because the sensor nodes need to know their locations in order to detect and record events so that their data is meaningful. Manual assignment of node coordinates is one possibility, but is often impractical or impossible due to the number of nodes or method of deployment. Equipping each sensor with a GPS receiver is another solution, however it is often cost prohibitive in terms of both hardware and power requirements. Furthermore,

T. Eren and P. Belhumeur are supported by the National Science Foundation under grants NSF ITR IIS-03-25864, and NSF IIS-03-08185.

W. Whiteley is supported by the Natural Science and Engineering Research Council (Canada) and the National Institutes of Health (USA).

T. Eren is with the Department of Electrical Engineering, Kirikkale University, Turkey. tolga.eren@aya.yale.edu

W. Whiteley is with the Department of Mathematics and Statistics, York University, Toronto, Ontario, Canada. whiteley@mathstat.yorku.ca

P. N. Belhumeur is with the Department of Computer Science, Columbia University, New York, NY 10027 USA. belhumeur@cs.columbia.edu since GPS requires line-of-sight between the receiver and satellites, it may not work well in buildings or in the presence of obstructions such as dense vegetation, buildings, or mountains blocking the direct view to the GPS satellites. There is usually a sparse set of nodes, called anchor nodes, that have their world coordinates from either GPS or manual configuration.

There has been research in using mobile ad hoc networks for multi-robot systems. Such networks are formed by robots establishing communication links whenever possible. Robots become nodes and can act as routers in this type of network to pass information between robots which might not otherwise be able to communicate, hence more information is provided to robots about the system. Sharing local information with the rest of the system to improve performance in the deployment of robots as sensor networks, and on facilitating multi-robot systems has been a motive behind the research that specifically investigates the application of ad hoc networks to mobile robots. These multiple robot platforms are dynamic so that they can split or merge with other networks over time.

Pure distance information is used extensively in studying sensor networks and formations of robotic agents [1], [2], [3], [4], [5]. Rigidity of formations with distance information is well understood in 2-space and there are partial results in 3-space. Bearings along with distances have been used for navigation, surveying, and localization in single robot applications. The use of bearings in sonar and radar applications are decades old. Although the bearing resolution of sonar systems is improving rapidly, computer vision technology tends to have greater resolution than sonar in bearing angle. In multi-agent applications, bearings are used in robot formations and in sensor networks [6]. There is a concept termed 'parallel drawings' [7] which can assist analysis of the rigidity of formations where there is bearing data, and this paper draws on this tool, carrying further preliminary results that were published in [8].

For ad hoc sensor networks, localization has been addressed by many authors (see references in [1] and [9]). Approaches vary in the sensor wavelength that is in use such as optical, radio frequency (RF), ultrasound, or acoustic. Computation methods fall into three classes: centralized (only one node computes), locally centralized (some nodes with unknown positions compute), fully distributed (each node with unknown position computes). Approaches differ in the problem formulation (deterministic versus probabilistic), propagation assumptions, and the assumed density of anchor nodes with known location. 
Received signal strength (RSS) is available from the RF communications typically resident on a node; however there are high variability of propagation losses in RSS. The RSS is usually quantized to a single bit to indicate proximity. Ultrasonic and acoustic transducers have been used to measure time difference of arrival (TDoA), exploiting the relatively slow propagation speeds, and to measure angle of arrival using an array of microphones. Sub-meter accuracy is available from ultrasonic or acoustic measurements, however these modalities require additional hardware and may compromise stealth.

The term bearing refers to an angle measurement with respect to another object. In our case, the angle of arrival (AoA) capability provides for each node bearings to neighboring nodes with respect to a nodes own axis. There are a couple of ways that sensors measure AoA. One is phase interferometry: the angle is estimated by phase differences in the signal received by two or more individual sensors (microphones for acoustic signals or antennas for RF signals). With directional antennas, AoA estimation uses the RSS ratio between two (or more) directional antennas located on the sensor. Two directional antennas pointed in different directions, such that their main beams overlap, can be used to estimate the AoA from the ratio of their individual RSS values.

By providing information about the direction to neighboring sensors rather than the distance to neighboring sensors, bearing is a technique for determining the direction of propagation of a radio-frequency wave or an acoustic signal incident on an antenna array (microphones for acoustic signals) [10], [6]. Bearing information is used in the geolocation of cell phones to comply with regulations that require cell systems to report the location of a cell phone placing an emergency call. The AoA of the cell phone's signal from multiple base stations would be combined to determine the phone's location on the earth. In formations of mobile robotic agents, bearings have been used extensively to maintain formations [11].

All the approaches for measuring bearings require multiple sensor array elements, which can contribute to sensor device cost and size. However, acoustic sensor arrays may already be required in devices for many environmental monitoring and security applications, in which the purpose of the sensor network is to identify and locate acoustic sources. Locating the sensors themselves using acoustics in these applications is a natural extension. RF antenna arrays imply large device size unless center frequencies are very high. However, available bandwidth and decreasing manufacturing costs at millimeterwave frequencies may make them desirable for sensor network applications.

A drawback of AoA is that highly coherent receiver is needed, i.e., all channels must have the same effect on the received signal. Moreover, the cost of the receiver increases as the array size increases. The size should be reduced as much as possible, but, the number of elements required to obtain a given accuracy strongly depends on the radio environment.
Uniqueness of network localization solutions is addressed by the theory of rigid graphs. The theory of rigid graphs is also used in maintaining rigid formations of robotic agents. For distance measurements, a key insight from the theory is that sufficiently high connectivity guarantees, with high probability, a unique solution and computational complexity that scales only linearly with the number of nodes. Directions, bearings, angles in undirected formations were studied in [8]. The graph rigidity problem for undirected formations with bearings is the dual of the distance case. However, there exists no complete theory for point formations based solely on angles. Yet, with sufficient connectivity and one fixed direction, the bearing results may be applied to the abstract graph of links as vertices and angles as edges [12].

Rigidity in directed formations of mobile autonomous agents was studied in [13], [14], [15], [16], [17], [18]. Localization in robotic networks was studied in [11] using kinematics of linear mechanisms. In this paper, we derive the conditions for unique network localization by using rigidity and global rigidity of digraphs that represent the network topologies in which links are directed and bearing information is used. Graph theoretic results prove to be valuable as they only depend on the interconnection structure of the links without a need for actual positions of nodes. We will restrict our attention to networks in 2-space. A sequel paper will provide the results for 3 -space.

The paper is organized as follows. In $\S I I$, we start with the problem statement of network localization and defining point formations and rigidity, which will be used throughout the paper. We then present parallel rigidity of formations using parallel drawings in $\S I I I$. We investigate directed parallel rigidity in $\S \mathrm{IV}$. Finally, concluding remarks are given in $\S \mathrm{V}$.

\section{Network LOCALIZATION AND Formations}

\section{A. Formulation of Network Localization}

The interconnection structure of sensing/communication links between nodes is called network topology and is denoted with $\mathbf{N}$. Consider a network $\mathbf{N}$ in real 2-space consisting of a set of $m>0$ nodes labelled 1 through $m$ that represent special "anchor" nodes together with $n-m>0$ additional nodes labelled $m+1$ through $n$ that represent ordinary nodes. Each node is located at a fixed position in $\mathbb{R}^{2}$. Anchor nodes have GPS capabilities, thus they know their world coordinates. We will use the term heading with the meaning of bearing to north. In this paper, we assume that either all nodes have compass capabilities, or some nodes do have and others can infer heading information from their neighbors. (A sequel paper will deal with the case in which compasses are either not available, or biased by local conditions.) An example of propagation of heading information between nodes is given in [6]. Before going into detail, it is useful to formally state the network localization problem.

1) Problem Statement:: The 2-dimensional network localization problem is the determination of $n-m$ unknown node locations $p=\left\{p_{m+1}, p_{2}, \ldots, p_{n}\right\}$ given the known anchor locations $\left\{p_{1}, p_{2}, \ldots, p_{m}\right\}$, and measurements $\left\{M_{i, j}\right\}$, 
where $M_{i, j}$ is a measurement by node $i$ related to any physical reading that indicates distance, or bearing by using a signal coming from node $j$. We do not assume full measurements, so we define the set $\mathcal{N}(i)$ to be the set of sensors with which sensor $i$ makes measurements. Clearly, $i \notin \mathcal{N}(i)$, and $\mathcal{N}(i) \subset\{1, \ldots, n\}$. Note that these measurements could be attained via different modalities, e.g., RF, infrared (IR), acoustics, or a combination of these.

\section{Neighbor relationship}

Although a node's "neighbors" are typically defined to be all other nodes within some specified range, other definitions could also be used. Ideally, sensors are assumed to have sensing regions in circular shape. Let $i$ and $j$ be two nodes in the network and $d(i, j)$ be the Euclidean distance between them. Let also $r(i)$ and $r(j)$ be the transmitting range of nodes $i$ and $j$, assuming the ideal case where coverage zones are perfect disks. If there exist two nodes $i, j$ in the network such that $\min [r(i), r(j)]<d(i, j)$ and $\max [r(i), r(j)]>$ $d(i, j)$, then $\mathbf{N}$ has directed links. In practice, the sensing region of a sensor is not necessarily a circle. In most cases, it is location-dependent and likely irregular. Thus, an inherent characteristic of wireless networks relies on the directionality of the associated graph. Directed graphs are likely to occur in scenarios where nodes perform independent transmitting range control or have heterogeneous terminals [19], [20]. Then implementing an efficient distributed topology control is crucial. In practice, this depends on the underlying protocols [20]. In robot networks, localization with directed graphs was studied in [11].

If we assume a centralized computation, with a supervisor that has access to all the data, the directed constraints just become constraints (linear equations without direction). The computation of the location is then direct. As it will become clear later, the calculation with directions is solving linear equations, with a one-parameter solution space, up to translation of the first node. This parameter can be scaled to make any single edge the desired length. Now, if we want fully distributed computation of locations by the nodes, then directed link matters a lot. The essential property we require in this paper is that the neighbor relationship is not necessarily a symmetric relation on $\{1,2, \ldots, n\}$. In this paper, we use the following definition: Given a node $i$, any node that is in the sensing region of $i$ is called a neighbor of $i$. It is possible that while node $j$ is in the sensing region of node $i$, vice versa may not be true.

Under these conditions $\mathrm{N}$ 's neighbor relationships can be conveniently described by a digraph $\mathbb{G}_{\mathbf{N}}=(\mathcal{V}, \mathcal{E})$ with vertex set $\mathcal{V}=\{1,2, \ldots, n\}$ where the elements of $\mathcal{V}$ denote the labels of nodes, and $\mathcal{E}$ is the set of ordered pairs of vertices called directed edges defined so that $(i, j)$ is one of the graph's edges precisely when node $j$ is in the sensing region of node $i$. A graph in which each edge is replaced by a directed edge is called a digraph. A digraph having no multiple edges or loops (corresponding to a binary adjacency matrix with 0's on the diagonal) is called a simple digraph. We assume throughout that $\mathbb{G}_{\mathbf{N}}$ is a connected digraph. The network localization problem is to determine the locations $p_{i}$ of all nodes in $\mathbb{R}^{2}$ given the graph of the network $\mathbb{G}_{\mathbf{N}}$, the positions of the anchor nodes $p_{j}, j \in\{1,2, \ldots, m\}$ in $\mathbb{R}^{2}$, and the measurements $M_{\mathbf{N}}(i, j)$ in $E_{\mathbf{N}}$. The network localization problem is generically solvable at $\left\{p_{1}, p_{2}, \ldots, p_{n}\right\}$ if it is solvable at each point in an open neighborhood of $\left\{p_{1}, p_{2}, \ldots, p_{n}\right\}$. To be more precise, generic points are defined as follows. A set $\mathcal{A}=\left(\alpha_{1}, \ldots, \alpha_{m}\right)$ of distinct real numbers is said to be algebraically dependent if there is a non-zero polynomial $h\left(x_{1}, \ldots, x_{m}\right)$ with integer coefficients such that $h\left(\alpha_{1}, \ldots, \alpha_{m}\right)=0$. If $\mathcal{A}$ is not algebraically dependent, it is called generic [21]. We say that $p=$ $\left(p_{1}, \ldots, p_{n}\right)$ is generic in 2 -space, if its $2 n$ coordinates are generic. It can be shown that the set of generic $p$ 's form an open dense subset of $\mathbb{R}^{2 n}$.

To study the solvability of the network localization problem, we reformulate the problem in terms of a "point formation." The point formation relevant to the network localization problem has associated with it the grounded graph of the network, $\hat{\mathbb{G}}_{\mathbf{N}}$, with the same vertices as $\mathbb{G}_{\mathbf{N}}$ but with a slightly larger edge set which adds "links" or edges from every anchor node to every other [1]. It is a property of $\hat{\mathbb{G}}_{\mathbf{N}}$ rather than $\mathbb{G}_{\mathbf{N}}$ which proves to be central to the solvability of the localization problem under consideration.

\section{B. Point Formations}

We begin by reviewing the point formation concept. By a 2 -dimensional point formation [1] at $p \triangleq\left[p_{1}, p_{2}, \ldots, p_{n}\right]^{T}$, written $\mathbb{F}_{p}$, is meant a set of $n$ points $\left\{p_{1}, p_{2}, \ldots, p_{n}\right\}$ in $\mathbb{R}^{2}$ together with a set $\mathcal{E}$ of $k$ links, labelled $(i, j)$, where $i$ and $j$ are distinct integers in $\{1,2, \ldots, n\}$. In this context, the points $p_{i}$ represent the positions of nodes (i.e., both anchor nodes and ordinary nodes), in $\mathbb{R}^{2}$ and the ordered pairs in $\mathcal{E}$ label those specific ordered node pairs between which there is a physical reading. Specifically, we denote the set of links with distance measurements by $\mathcal{L}$, the one with bearing measurements with $\mathcal{B}$. For the network $\mathbf{N}, \mathcal{E}$ would consist of all edges in $\hat{\mathbb{G}}_{\mathbf{N}}$, since the distance between every pair of anchor nodes is determined by their specified positions. In a network in which nodes have only distance and bearing measurements, $\mathcal{E}$ is the union of $\mathcal{L}, \mathcal{B}$ and the set of implicit links among anchor nodes.

A definition of global rigidity for networks with distance measurements was given in [1]. Here we generalize this definition to include other types of physical measurements, e.g., direction, angle, bearing. Each point formation $\mathbb{F}_{p}$ uniquely determines a graph $\mathbb{G}_{\mathbb{F} p} \triangleq\{\mathcal{V}, \mathcal{E}\}$ with vertex set $\mathcal{V} \triangleq\{1,2, \ldots, n\}$ and edge set $\mathcal{E}$, as well as a measurement function $f: \mathcal{L} \mapsto \mathbb{R}$ (for distances), $h: \mathcal{B} \mapsto[0,2 \pi)$ (for bearings) whose value at $(i, j) \in \mathcal{E}$ is the measured quantity (distance, angle, etc.) between $p_{i}$ and $p_{j}$. Let us note that the measurement function of $\mathbb{F}_{p}$ is the same as the measurement function of any point formation $\mathbb{F}_{q}$ with the same graph as $\mathbb{F}_{p}$ provided $q$ is congruent to $p$ in the sense that there is a distance preserving map $T: \mathbb{R}^{2} \rightarrow \mathbb{R}^{2}$ such that $T\left(q_{i}\right)=p_{i}, i \in\{1,2, \ldots, n\}$. We say that two point formations $\mathbb{F}_{p}$ and $\mathbb{F}_{q}$ are congruent if they have the same 
graph and if $q$ and $p$ are congruent. It is clear that $\mathbb{F}_{p}$ is uniquely determined by its graph and measurement function at most up to a congruence transformation. A formation that is exactly determined up to congruence by its graph and measurement function is called "globally rigid."

Now we focus on rigidity which is closely related to global rigidity. Rigidity has been used extensively for mobile formations [2], [3], [8]. Let us imagine a point formation moving in real 2 -space. A point formation is called rigid if the distance between each pair of nodes does not change over time under ideal conditions. In reality, nodes are entities with physical dimensions. For modelling purposes, nodes are represented by points called point nodes. A point node with an attached coordinate system is called an oriented node. A graph $\mathbb{G}=(\mathcal{V}, \mathcal{E})$ is called generically rigid, if $\mathbb{F}(p)=(p, \mathcal{E})$ is rigid for a generic $p$. The property of generic rigidity does not depend on the precise distances between the points of $\mathbb{F}(p)$ but predicts the rigidity of a formation from the graph of the vertices and links, in other words, by the underlying graph.

Distances between all node pairs can be held fixed by directly measuring distances between only some nodes and keeping them at desired values. A 'distance constraint' is a requirement that a distance between between two nodes, depicted with $d$, be maintained through a sensing/communication link and some control strategy. For example, a distance constraint between two nodes, depicted with $d_{i j}$, is shown in Figure 1(a). Distance constraints are sometimes referred to as range or separation constraints. With enough distance constraints, the whole formation will be rigid, even without there being a distance constraint between every pair of nodes.

Another form of constraint is a 'bearing constraint', and such constraints, generally in conjunction with distance constraints, can contribute to establishing rigidity. A bearing is the angle between a sensing/communication link and the $x$ axis of a node's local coordinate system. For example, if two nodes $i$ and $j$ have a sensing/communication link between each other as shown in Figure 1(a), then bearing constraints for $i$ and $j$, denoted by $\theta_{i j}$ and $\theta_{j i}$ respectively, are the angles between the $x$-axis of each node's local coordinate system and the link $(i, j)$. As it will become clear later, bearing information can be used by both nodes in a formation with symmetric neighbor relation, i.e., node $i$ measures $\theta_{i j}$ and node $j$ measures $\theta_{j i}$ concurrently. Alternatively, bearing information can be used by only one of the nodes in a formation with directed links, i.e., either node $i$ measures $\theta_{i j}$ or node $j$ measures $\theta_{j i}$. Our aim is to obtain a relation between the coordinates of node $i$ and $j$ given the bearing constraint between them. In real implementations of bearing information, the information about a global coordinate system $\left(x_{G}, y_{G}\right)$ is either known by all nodes or is transmitted from anchor nodes to ordinary nodes. This is done by passing "heading" information from one node to another. By heading is meant the angle between the $y$-axis of the global coordinate system and the $x$-axis of the node's local coordinate system. For example, $\phi_{i}$ is the heading of $i$ in

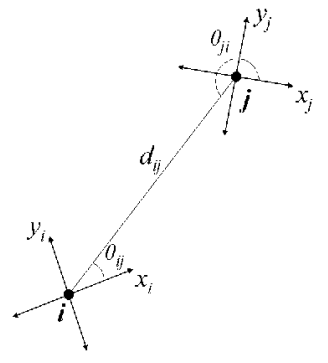

(a)

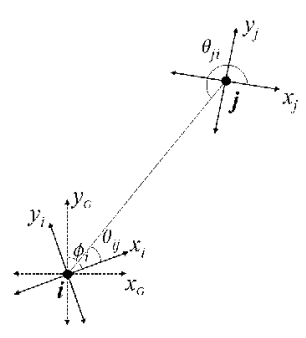

(b)

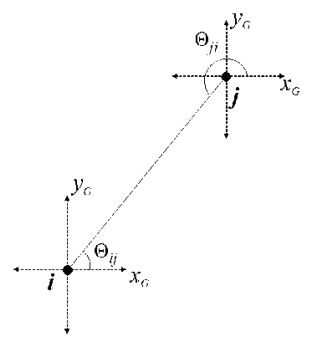

(c)

Fig. 1. (a) Bearing constraints for $i$ and $j$ are denoted by $\theta_{i j}$ and $\theta_{j i}$, respectively; (b) $\phi_{i}$ is the heading of $i$; (c) Once nodes know the global coordinate system, they can transform the bearing information measured in their local coordinate systems $\left(\theta_{i j}\right.$ and $\left.\theta_{j i}\right)$ into bearing information in the global coordinate system $\left(\Theta_{i j}\right.$ and $\left.\Theta_{j i}\right)$.

Figure 1(b). Once node $i$ passes the information $\phi_{i}$ and $\theta_{i j}$ to node $j$, then node $j$ can compute its heading by $\phi_{j}=$ $\pi-\left(\theta_{i j}-\phi_{i}\right)+\theta_{j i}$. Once nodes know the global coordinate system, they can transform the bearing information measured in their local coordinate systems $\left(\theta_{i j}\right.$ and $\left.\theta_{j i}\right)$ into bearing information in the global coordinate system $\left(\Theta_{i j}\right.$ and $\left.\Theta_{j i}\right)$ as shown in Figure 1(c). We note that $\Theta_{j i}=\pi+\Theta_{i j}$.

\section{Parallel Rigidity}

Before proceeding further, we introduce "parallel drawings." Parallel drawings have been studied in rigidity and plane configurations in computer-aided design (CAD). They are particularly relevant when the configurations being considered are constrained using bearing-only information, as will become clear later. A plane configuration is a collection of geometric objects such as points, line segments, and circular arcs in the plane, together with constraints on and between these objects. Two point formations on the same graph are parallel drawings if corresponding edges are parallel. Parallel drawings, used by engineering draftsmen in the nineteenth century, have reappeared in a number of branches of discrete geometry [22].

Given a point formation $\mathbb{F}_{r}$, we are interested in parallel drawings $\mathbb{F}_{s}$ in which $s_{i}-s_{j}$ is parallel to $r_{i}-r_{j}$ for all $(i, j) \in \mathcal{E}$. Using the operator $(.)^{\perp}$, for turning a plane vector by $\frac{\pi}{2}$ counterclockwise, these constraints can be written:

$$
\left(r_{i}-r_{j}\right)^{\perp} \cdot\left(s_{i}-s_{j}\right)=0 .
$$

Each such constraint is called a direction constraint in CAD literature. This gives a system of $|\mathcal{E}|$ homogeneous linear equations, and a parallel drawing is a solution of this system. 
We have the following proposition.

Proposition 3.1: A bearing constraint can be written as a parallel drawing constraint.

Proof: A bearing constraint for node $i$ along the trajectory $q$ can be expressed as:

$$
\measuredangle\left[\left(q_{j}(t)-q_{i}(t)\right), e_{x}\right]=\Theta_{i j}
$$

and similarly the bearing constraint for node $j$ along the trajectory $q$ can be expressed as:

$$
\measuredangle\left[\left(q_{i}(t)-q_{j}(t)\right), e_{x}\right]=\Theta_{j i}
$$

where $e_{x}$ is the unit vector along the $x$-axis of the global coordinate system, and $\measuredangle[$.$] stands for the function that$ maps the two vectors in the argument to the angle between them, where the angle is measured in the counterclockwise direction from the second vector to the first vector in the argument. Let us consider a particular fixed set of points, $p_{d}=\left(p_{1}, p_{2}, \ldots, p_{n}\right)$, along the trajectory $q(t)$ where the bearing constraints are satisfied. We can think of $p_{d}$ as a reference set of points that determines the desired bearing constraints for the formation and the nodes can be thought of satisfying the bearing constraints set by $p_{d}$ at all other points along the trajectory. For node $i$, we can write

$$
\measuredangle\left[\left(p_{j}-p_{i}\right), e_{x}\right]=\Theta_{i j}
$$

and for node $j$, we get

$$
\measuredangle\left[\left(p_{i}-p_{j}\right), e_{x}\right]=\Theta_{j i} .
$$

Next, let us consider the vector $\left(p_{j}-p_{i}\right)^{\perp}$ which is obtained by rotating the vector $\left(p_{j}-p_{i}\right)$ counterclockwise by $90^{\circ}$ and the vector $\left(p_{i}-p_{j}\right)^{\perp}$ which is obtained by rotating the vector $\left(p_{i}-p_{j}\right)$ counterclockwise by $90^{\circ}$. For these two vectors we write,

$$
\measuredangle\left[\left(p_{j}-p_{i}\right)^{\perp}, e_{x}\right]=\frac{\pi}{2}+\Theta_{i j}(\bmod 2 \pi)
$$

and,

$$
\measuredangle\left[\left(p_{i}-p_{j}\right)^{\perp}, e_{x}\right]=\frac{\pi}{2}+\Theta_{j i}(\bmod 2 \pi)
$$

By (2) and (6), we can write

$$
\measuredangle\left[\left(p_{j}-p_{i}\right)^{\perp},\left(q_{j}(t)-q_{i}(t)\right)\right]=\frac{\pi}{2}
$$

and by (3) and (7), we can write

$$
\measuredangle\left[\left(p_{i}-p_{j}\right)^{\perp},\left(q_{i}(t)-q_{j}(t)\right)\right]=\frac{\pi}{2} .
$$

(10) and (9) imply

$$
\left(p_{i}-p_{j}\right)^{\perp} \cdot\left(q_{i}(t)-q_{j}(t)\right)=0 .
$$

For every link with a bearing constraint in the point formation, it is now straightforward to write

$$
\left(p_{i}-p_{j}\right)^{\perp} \cdot\left(q_{i}(t)-q_{j}(t)\right)=0, \quad(i, j) \in \mathcal{B}, \quad t \geq 0 .
$$

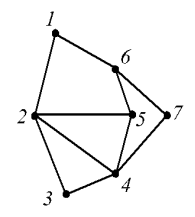

(a)

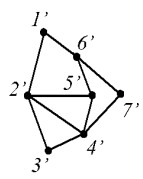

(c)

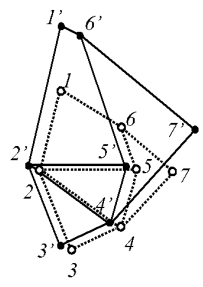

(e)

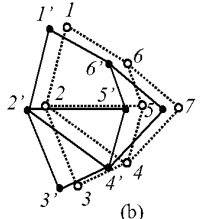

(b)

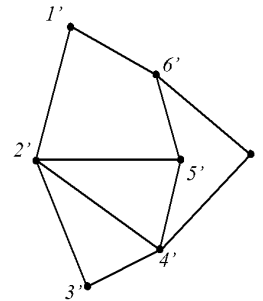

(d)

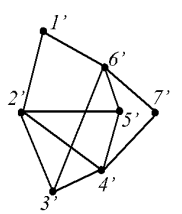

(f)
Fig. 2. Parallel point formations.

This gives a system of $|\mathcal{B}|$ homogenous linear equations. A solution of this system is called a parallel point formation.

Central to the development in the rest of this section will be the use of parallel drawings of configurations [23], [24], [25]. Given a point formation in 2-space with bearing constraints $\mathbb{F}_{p}$, we are interested in parallel point formations $\mathbb{F}_{r}$ in which $r_{i}-r_{j}$ is parallel to $p_{i}-p_{j}$ for all $(i, j) \in \mathcal{B}$. Trivially parallel point formations are translations and dilations of the original point formation, including the parallel point formation in which all points are coincident. All others are non-trivial. For example, Figure $2 b$ shows a translation of the point formation in Figure 2a; and Figure 2c and Figure $2 d$ are dilations of the point formation in Figure 2a. In particular Figure $2 \mathrm{c}$ is a contraction and Figure $2 \mathrm{~d}$ is an expansion. Figure 2e shows a non-trivial parallel point formation of Figure 2a because the point formation in Figure 2e cannot be obtained from the point formation in Figure 2a by translation or dilation although all the corresponding links in these two point formations are parallel to each other $\left((i, j)\right.$ and $\left(i^{\prime}, j^{\prime}\right)$ are corresponding links). A point formation with bearing constraints is called parallel rigid if all parallel point formations are trivially parallel. Otherwise it is called flexible. For example, the point formation in Figure $2 \mathrm{a}$ is flexible. On the other hand, the point formation in Figure $2 \mathrm{f}$, which is obtained from the point formation in Figure 2a by inserting an extra link $\left(3^{\prime}, 6^{\prime}\right)$, is a parallel rigid point formation.

Taking the derivative of (11) (recall that $p$ is a fixed point set and $q(t)$ is time varying in (11)), we obtain 


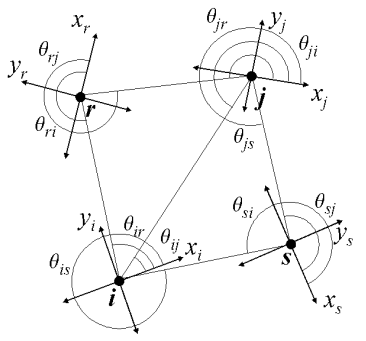

(a)

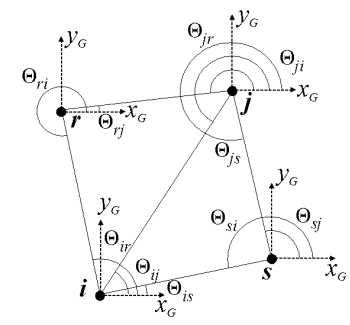

(b)
Fig. 3. Consider a planar point formation $\mathbb{F}_{p}$ with bearing constraints shown in (a). We assume that at least one node knows the global coordinate system and the information about this global coordinate system is passed to the other nodes in the formation. The same point formation drawn with bearing constraints in the global coordinate system is shown in (b).

$$
\left(p_{i}-p_{j}\right)^{\perp} \cdot\left(\dot{q}_{i}(t)-\dot{q}_{j}(t)\right)=0, \quad(i, j) \in \mathcal{B}, \quad t \geq 0
$$

These equations can be rewritten in matrix form as

$$
R_{\mathcal{B}}(p) \dot{q}=0
$$

where $\dot{q}=\left[\dot{q}_{1}, \dot{q}_{2}, \ldots, \dot{q}_{n}\right]^{T}$ and $R_{\mathcal{B}}(p)$ is the rigidity matrix for formations with bearing information.

It is shown in [26] that any statement for a point formation of distances can be given for the same point formation of directions where distances are switched with directions. The isomorphism goes down the pairs of columns for each vertex, turning all the vectors by $90^{\circ}$ (in a direction of choice). This process preserves the solution space (just turning the solutions by $90^{\circ}$ as well), and turns each row for a distance into a row for a direction. Because of this geometric switching, there is a generic switching theorem in [26] that converts results in a direct fashion, so the generic type of rigidity is defined in the same manner as in the case of distances. Thus the graph theoretic test is given with the following theorem:

Theorem 3.1: A graph $\mathbb{G}=(\mathcal{V}, \mathcal{B})$ is generically parallel rigid in 2-space if and only if there is a subset $\mathcal{B}^{\prime} \subseteq \mathcal{B}$ satisfying the following two conditions: (1) $\left|\mathcal{B}^{\prime}\right|=2|\mathcal{V}|-3$, (2) For all $\mathcal{B}^{\prime \prime} \subseteq \mathcal{B}^{\prime}, \mathcal{B}^{\prime \prime} \neq \emptyset,\left|\mathcal{B}^{\prime \prime}\right| \leq 2\left|\mathcal{V}\left(\mathcal{B}^{\prime \prime}\right)\right|-3$, where $\left|\mathcal{V}\left(\mathcal{B}^{\prime \prime}\right)\right|$ is the number of vertices that are end-vertices of the edges in $\mathcal{B}^{\prime \prime}$.

For networks using pure distance information, the conditions for global rigidity are stronger than those for rigidity [1]. For networks in 2-space with bearing information between nodes, the situation is strikingly different. Because the key constraints are linear equations, if there are two nonsimilar parallel formations with points $p$ and $q$, then both formations are not rigid. Therefore, for these formations, rigidity implies global rigidity up to similarity. In 2-space, if we have the $2 n-3$ bearings of a parallel rigid formation, and add one length, we will have a globally rigid formation. We do have a simple combinatorial characterization (counting) and fast algorithms for global rigidity.

Theorem 3.2: If $\mathbb{F}_{p}$ is a formation in 2-space, then $\mathbb{F}_{p}$ is parallel rigid if and only if $\mathbb{F}_{p}$ is globally rigid under translation and dilation maps.
Proof: Suppose that $\mathbb{F}_{p}$ is not globally rigid. Therefore, there is a parallel drawing $\mathbb{F}_{q}$ which is not similar to $\mathbb{F}_{p}$ as a configuration. We will show that $\mathbb{F}_{p}$ is flexible with $\mathbb{F}_{q}$ as a non-trivial parallel drawing. For all edges $(i, j) \in \mathcal{B},\left(p_{i}-p_{j}\right)$ is parallel to $\left(q_{i}-q_{j}\right)$. Therefore, $\left(p_{i}-p_{j}\right)^{\perp} \cdot\left(q_{i}-q_{j}\right)=0$ as required. Since $\mathbb{F}_{p}$ is not similar to $\mathbb{F}_{q}$, there is some pair $(h, k) \notin \mathcal{B}$ such that $p_{h}-p_{k}$ is not parallel to $q_{h}-q_{k}$. Therefore, $\left(p_{h}-p_{k}\right)^{\perp} \cdot\left(q_{h}-q_{k}\right) \neq 0$. This confirms that $\mathbb{F}_{q}$ is a non-trivial parallel drawing of $\mathbb{F}_{p}$.

Conversely, suppose that $\mathbb{F}_{p}$ is flexible with a non-trivial parallel drawing $\mathbb{F}_{q}$. Then $\mathbb{F}_{q}$ itself is the non-similar parallel drawing of $\mathbb{F}_{p}$ which shows it is not globally rigid.

More generally, nodes are not confined to use their sensing and communication links for measuring distances only. We can exploit such a possibility to generate point formation that is not only locally unique but also globally unique with as few links as a minimally rigid formation.

For formations with combined distance-bearing constraints, there is the following combinatorial characterization of parallel rigidity.

Theorem 3.3: With $\mathcal{L}$ for distances and $\mathcal{B}$ for bearings, a graph $\mathbb{G}=(\mathcal{V}, \mathcal{L}, \mathcal{B})$ is generically parallel rigid in 2-space if and only if the following conditions hold: (i) $|\mathcal{L} \cup \mathcal{B}|=2|\mathcal{V}|-$ 2; (ii) for all subsets $\mathcal{V}^{\prime}$ of vertices: $\left|\mathcal{L}^{\prime} \cup \mathcal{B}^{\prime}\right| \leq 2\left|\mathcal{V}^{\prime}\right|-2$; (iii) for all subsets $\mathcal{V}^{\prime}$ of at least two vertices: $\left|\mathcal{B}^{\prime}\right| \leq 2\left|\mathcal{V}^{\prime}\right|-3$ (iv) for all subsets $\mathcal{V}^{\prime}$ of at least two vertices: $\left|\mathcal{L}^{\prime}\right| \leq 2\left|\mathcal{V}^{\prime}\right|-3$.

The proof for the characterization of parallel rigidity for distance-direction constraints is given in [23]. Since it is closely related to parallel rigidity for distance-bearing constraints, the proof is omitted here.

This characterization also covers the distance-bearing combinations that permit more than one distance. Note that this is the criterion for rigidity up to translation, not for global rigidity, if there are multiple distance constraints. We suspect that it is probably the criterion for global rigidity, up to translation, if there are enough bearing constraints. Under this assumption, and assuming that angle of arrival is measured in trigonometric direction, we have a conjecture for global rigidity. But before that, we need a few auxiliary definitions. A tree is a graph in which any two vertices are connected by exactly one path. Given a connected, undirected graph, a spanning tree of that graph is a subgraph which is a tree and connects all the vertices together.

Conjecture 3.1: Provided that the bearing constraints form at least a spanning tree, then for generic formations, rigidity up to translation is equivalent to global rigidity of the formation.

\section{DiRected PARAllel Rigidity}

A directed edge is written with an ordered pair of endvertices $(i, j)$ representing an edge directed from $j$ to $i$ and drawn with an arrow from $j$ to $i$, that is from the source to the sink. The number of edges directed into a given vertex $i$ in a digraph $\mathbb{G}$ is called the in-degree of the vertex and is denoted by $d_{\mathbb{G}}^{-}(i)$. The number of edges directed out from a given vertex $i$ in a digraph $\mathbb{G}$ is called the out-degree of the vertex and is denoted by $d_{\mathbb{G}}^{+}(i)$. The out-neighborhood 
$N_{\mathbb{G}}^{+}(i)$ of a vertex $i$ is $\{j \in \mathcal{V}:(j, i) \in \mathcal{E}\}$, and the inneighborhood $N_{\mathbb{G}}^{-}(i)$ of a vertex $i$ is $\{j \in \mathcal{V}:(i, j) \in \mathcal{E}\}$. The union of out-neighborhood and in-neighborhood is the set of neighbors of $i$, i.e., the (open) neighborhood of $i$, $\mathcal{N}_{\mathbb{G}}(i)$. When $i$ is also included, it is the closed neighborhood of $i, \mathcal{N}_{\mathbb{G}}[i]$. A directed path is a sequence $\{i, j, k, \ldots, r, s\}$ such that $(i, j),(j, k), \ldots,(r, s)$ are directed edges of the graph. A cycle is a directed path such that the first vertex of the path equals the last. A digraph is acyclic if it does not contain any cycle.

Since parallel rigidity problem for formations with bearings is isomorphic to the distance case, we can make use of some of the techniques developed for formations with distance information. There are sequential steps to create directed rigid formations developed in [14], [15]. Then, one operation for extending a parallel rigid graph is directed vertex addition: given a minimally rigid graph $\mathbb{G}^{*}=\left(\mathcal{V}^{*}, \mathcal{B}^{*}\right)$, we add a new vertex $i$ of out-degree 2 with two edges directed from $i$ to two other vertices in $\mathcal{V}^{*}$. The second operation preserving directed parallel rigidity is directed edge splitting: given directed parallel rigid graph $\mathbb{G}^{*}=\left(\mathcal{V}^{*}, \mathcal{B}^{*}\right)$, we remove a directed edge $(j, k)$ (directed from $j$ to $k$ ) in $\mathcal{B}^{*}$ and then we add a new vertex $i$ of out-degree 2 and in-degree 1 with three edges by inserting two edges $(j, i),(i, k)$, and one edge between $i$ and one other vertex (other than $j, k$ ) in $\mathcal{V}^{*}$ such that the edge $(j, i)$ is directed from $j$ to $i$ and the other two edges are directed from $i$ to the other vertices. The third operation which will complete the constructions is edge reversal: edge $(a, b)$ is reversed to $(b, a)$, if for the in-vertex $b, \mathcal{N}_{\mathbb{G}}^{+}(b)<2$.

A directed Henneberg sequence for the graph $\mathbb{G}$, is a sequence of steps, starting from a single edge, using the following steps, and ending with the graph $\mathbb{G}$ :

1) directed vertex addition;

2) directed edge split;

3) edge reversal.

From the isomorphism between distances and bearings, and from Theorem 3.6 in [15], all possible directed parallel rigid graphs are generated by a directed Henneberg sequence. A digraph constructed by using only directed vertex addition is called a simple graph.

\section{2-directed digraphs}

Any ordinary node in a network with directed bearing links needs to have at least two in-coming links to localize itself, that is, $d_{\mathbb{G}}^{-}(i) \geq 2$. Of course, anchor nodes do not need any in-coming links to localize themselves. However, we insert the implicit links among anchor nodes to obtain the grounded graph. Furthermore, the existence of one anchor node rules out translation. If there is only one anchor node, then scaling is still allowed. This means that one ordinary node can have one degree of freedom. We call such a node a free node. Anchor node and free node together make up the set of guide nodes. The digraph $\mathbb{G}=(\mathcal{V}, \mathcal{E})$ is 2-directed if for all $i \in \mathcal{V}$, $d_{\mathbb{G}}^{-}(i) \leq 2$.

A point formation with directed bearing constraints is called directed parallel rigid if all directed parallel point formations are trivially parallel. There are a number of issues that must be addressed in the localization of networks with directed links. We identify three key layers as follows: 1) parallel rigidity of the underlying undirected formation; 2) directed parallel rigidity of the formation; 3) convergence and the quality of initial position estimates in the network. If underlying undirected formation is non-rigid, then directed formation cannot be rigid. Thus, undirected rigidity is a necessary condition for directed rigidity. On the other hand, when we associate a direction to each link in a rigid undirected formation, directed parallel rigidity is not necessarily guaranteed, because an in-degree of a node may be set to 1 while another node has an in-degree of 3 resulting from a poor selection of directions. Consequently, undirected parallel rigidity is not a sufficient condition for directed parallel rigidity. Even if there is subset of the grounded graph that is 2-directed, the undirected graph may still be flexible. Thus, we can state the conditions for global rigidity for a directed graph as follows: Given a grounded digraph $\hat{\mathbb{G}}=(\mathcal{V}, \hat{\mathcal{B}})$ of the network $\mathbf{N}$, there is a subset $\mathcal{B}^{\prime} \subseteq \hat{\mathcal{B}}$ satisfying the following conditions:

1) $\left|\mathcal{B}^{\prime}\right|=2|\mathcal{V}|-3$,

2) For all $\mathcal{B}^{\prime \prime} \subseteq \mathcal{B}^{\prime}, \mathcal{B}^{\prime \prime} \neq \emptyset,\left|\mathcal{B}^{\prime \prime}\right| \leq 2\left|\mathcal{V}\left(\mathcal{B}^{\prime \prime}\right)\right|-3$, where $\left|\mathcal{V}\left(\mathcal{B}^{\prime \prime}\right)\right|$ is the number of vertices that are end-vertices of the edges in $\mathcal{B}^{\prime \prime}$.

3) $\mathbb{G}^{\prime}=\left(\mathcal{V}, \mathcal{B}^{\prime}\right)$ is 2-directed.

In a fully distributed computation, the propagation of position/bearing information works as follows: Nodes immediately adjacent to an anchor node get their distance/bearings directly from the anchor node. Assuming that a node has some neighbors with distance/bearing information for an anchor node, it will be able to compute its own distance/bearing with respect to that anchor node, and forward it further into the network. Assuming that the conditions 1 through 3 above are met, convergence problems arise as the third layer, because initial position estimates by each node, delays, errors in measurements, may cause non-convergent solutions. Moreover, accumulation of errors in cycles arise in digraphs. 'Simple graphs' as described above are inherently much easier to work with to avoid convergence problems.

If all nodes have only bearing measurements and the formation is directed parallel rigid, at least one distance constraint is necessary to rule out scaling. Distance constraint can exist in the network in two scenarios: (i) at least one node measures distance; (ii) there are at least two anchor nodes in the network. The set of anchor nodes is denoted by $\mathcal{A}$. Let us note that the distance and bearing between every pair of anchor nodes are implicitly known.

Recall that there are absolute and relative localization. Let us consider absolute localization. If a formation with bearings is globally rigid, translations are still possible in a parallel rigid formation. We need at least one anchor node with world coordinates to pin down the whole network to exclude translations. Thus $|\mathcal{A}| \geq 1$. Furthermore, we need at least one distance information to rule out scaling. If $|\mathcal{A}| \geq 2$ then scaling is automatically excluded. If $|\mathcal{A}|=1$ then 
we need at least one ordinary node that has a distance measurement from its neighbor. For relative localization, anchor nodes are not necessary, and parallel rigidity and one distance constraint will be sufficient.

We summarize the conditions for network localization with bearings as follows: A network $\mathbf{N}$ is localizable (in the absolute sense) if the following conditions hold:

1) its underlying directed graph is globally rigid;

2) $|\mathcal{A}| \geq 1$.

3) there exists at least one distance constraint - this condition holds if either

- $|\mathcal{A}| \geq 2$; or,

- there exists at least one ordinary node that has a distance measurement from its neighbor.

A network $\mathbf{N}$ is localizable (in the relative sense) if the following conditions hold:

1) its underlying directed graph is globally rigid;

2) there exists at least one distance constraint - this condition holds if either

- $|\mathcal{A}| \geq 2$; or,

- there exists at least one ordinary node that has a distance measurement from its neighbor.

\section{CONCLUding REMARKS}

If there is more than one distance constraint in the network, then multiple realizations again become possible depending on the distribution of distance and bearing constraints.

A sequel will provide the results for the following types of networks:

- networks in both 2- and 3-space under the assumption that the information obtained by compasses is either not available, or biased by local conditions;

- networks with mixed bearings-distances or anglesdistances in both 2- and 3-space;

Finally, directed rigidity have been applied for coordinated motion of robotic agents in directed rigid formations (see for example [8], [14], [15], [16], [17], [18]). Our results on using bearing-angle information in mobile directed formations will be in a separate consecutive paper.

\section{REFERENCES}

[1] J. Aspnes, T. Eren, D. Goldenberg, W. Whiteley, Y. R. Yang, A. S. Morse, B. D. O. Anderson, and P. N. Belhumeur, "A theory of network localization," IEEE Transactions on Mobile Computing, 2006, to appear. [Online]. Available: http://www.cs.yale.edu/homes/dkg6/papers/TMC.pdf

[2] R. Olfati-Saber and R. Murray, "Distributed structural stabilization and tracking for formations of dynamic multi-agents," in Proceedings of the 41st IEEE Conference on Decision and Control, Las Vegas, NV, December 2002.

[3] J. Baillieul and A. Suri, "Information patterns and hedging Brockett's theorem in controlling vehicle formations," in Proceedings of the 42nd IEEE Conference on Decision and Control, Maui, Hawaii, December 2003.

[4] P. Tabuada, G. Pappas, and P. Lima, "Feasible formations of multiagent systems," in Proceedings of the American Control Conference, 2001, pp. 56-61.

[5] H. G. Tanner, G. J. Pappas, and V. Kumar, "Leader-to-formation stability," IEEE Transactions on Robotics and Automation, vol. 20, no. 3, pp. 443-455, June 2004.
[6] D. Niculescu and B. Nath, "Ad hoc positioning system (APS) using AoA," in Proceedings of IEEE INFOCOM '03, San Francisco, CA, April 2003.

[7] W. Whiteley, "Matroids from discrete geometry," in Matroid Theory, J. E. Bonin, J. G. Oxley, and B. Servatius, Eds. American Mathematical Society, Contemporary Mathematics, 1996, vol. 197, pp. 171-313.

[8] T. Eren, W. Whiteley, A. Morse, P. Belhumeur, and B. Anderson, "Sensor and network topologies of formations with direction, bearing and angle information between agents," in Proceedings of the 42nd IEEE Conference on Decision and Control, December 2003, pp. 3064 3069.

[9] N. Patwari, J. N. Ash, S. Kyperountas, A. O. Hero, R. L. Moses, and N. S. Correal, "Locating the nodes: cooperative localization in wireless sensor networks," IEEE Signal Processing Magazine, vol. 22, no. 4, pp. 54-69, July 2005.

[10] J. Ash and L. Potter, "Sensor network localization via received signal strength measurements with directional antennas," Proceedings of the Forty-Second Annual Allerton Conference on Communication, Control, and Computing, pp. 1861-1870, Champaign-Urbana, IL, Sep 2004.

[11] F. Zhang, B. Grocholsky, and V. Kumar, "Formations for localization of robot networks," in Proceedings of the 2004 IEEE International Conference on Robotics and Automation, April 2004.

[12] T. Eren, W. Whiteley, A. S. Morse, P. N. Belhumeur, and B. D. O. Anderson, "Sensor and network topologies of formations with direction, bearing and angle information between agents," in Proceedings of the 42nd IEEE Conference on Decision and Control, Maui, Hawaii, December 2003, pp. 3064-3069.

[13] T. Eren, W. Whiteley, B. D. O. Anderson, A. S. Morse, and P. N. Belhumeur, "Information structures to secure control of rigid formations with leader-follower architecture," in Proceedings of the American Control Conference, Portland, Oregon, June 2005, pp. 2966-2971.

[14] T. Eren, W. Whiteley, and P. Belhumeur, "Rigid formations with leader-follower architeture," Columbia University, Tech. Rep. CUCS-010-05, March 2005. [Online]. Available: http://www1.cs.columbia.edu/ library/

[15] _ "Rigid formations with leader-follower architeture," Columbia University, Tech. Rep. CUCS-008-06, December 2005. [Online]. Available: http://www1.cs.columbia.edu/ library/

[16] J. M. Hendrickx, B.D.O. Anderson, and V. D. Blondel, "Rigidity and persistence of directed graphs," in Proceedings of the 44th IEEE Conference on Decision and Control, and the European Control Conference, Seville, Spain, December 2005.

[17] C. Yu, B. Fidan, and B. D. O. Anderson, "Persistence acquisition and maintenance for autonomous formations," in Proceedings of Workshop on Unmanned Aerial Vehicles, 2nd International Conference on Intelligent Sensors, Melbourne, Australia, December 2005, pp. 379-384.

[18] B. Fidan and B. Anderson, "Acquiring and maintaining persistence of autonomous multi-vehicle formations," IEE Proc Control Theory and Applications, NICTA Ref PA5378.

[19] J. Blum, M. Ding, A. Thaeler, and X. Cheng, "Connected dominating set in sensor networks and MANETs," in Handbook of Combinatorial Optimization, D.-Z. Du and P. Pardalos, Eds. Kluwer Academic Publishers, 2004, pp. 329-369.

[20] P. Gober, A. Ziviani, P. Todorova, M. D. de Amorim, P. Hueneberg, and S. Fdida, "Topology control and localization in wireless adhoc and sensor networks," Ad Hoc \& Sensor Wireless Networks: An International Journal, vol. 1, no. 2, pp. 301-321, March 2005.

[21] R. Connelly, "Generic global rigidity," Discrete Comput. Geom., vol. 33, no. 4, pp. 549-563, April 2005.

[22] W. Whiteley, "Rigidity and scene analysis," in Handbook of Discrete and Computational Geometry, J. Goodman and J. O'Rourke, Eds. CRC Press, 1997, pp. 893-916.

[23] B. Servatius and W. Whiteley, "Constraining plane configurations in Computer Aided Design: Combinatorics of directions and lengths," SIAM Journal of Discrete Mathematics, vol. 12, pp. 136-153, 1999.

[24] W. Whiteley, "Parallel redrawing of configurations in 3-space," 1986, preprint.

[25] _ "A matroid on hypergraphs, with applications to scene analysis and geometry," Discrete and Computational Geometry, vol. 4, pp. 7595, 1988.

[26] — "Constraining plane configurations in CAD: geometry of lengths and directions," 1997, preprint. 